\title{
Semi-empirical Formulas and Numerical Tables for the Peripheral Jet of the ACVs
}

\author{
Hideo Matsuo*, Member
}

\begin{abstract}
Summary
Semi-empirical formulas previously proposed by the present author to estimate the characteristics of the peripheral jet of $\mathrm{ACV}_{\mathrm{s}}{ }^{* *}$ are re-examined for wider range of operating conditions and jet discharge angles and it has turned out that the proposed method is applicable with slight corrections. Numerical calculations are made for possible cases in the practical operation and the results are given in tabular forms.
\end{abstract}

\section{Introduction}

The analysis of the cushion stability of ACV is usually made quasi-statically and this requires investigation of unbalanced peripheral jets, viz., overfed and underfed jets. Many theories have been proposed for this purpose. However, most of them are based on the inviscid assumption, which leads to the conclusion that some non-linearity appears at the balanced point. In the previous paper ${ }^{1)}$, the author proposed empirical formulas which were derived from the theoretical consideration on the viscous jet, and ascertained the applicability of the formulas to the peripheral jet with discharge angle of $30^{\circ}$. It was also demonstrated there that the discontinuity did not occur in actual viscous jet. In the present study, further experiments are made to cover general operating conditions and jet discharge angle. Numerical calculations are made for possible cases and the results are given in tabular forms, by use of which practical estimation of jet characteristics will be made. Although we deal with the peripheral jet of ACV in this paper, the results will apply to other types of fluid suspension system with peripheral jets, viz., air cushion bearings, air cushion load carriers, etc.

\section{List of Symbols}

$\theta \quad$ Jet discharge angle (measured from horizontal downward)

$t \quad$ Nozzle thickness

$h$ Hoverheight

$\rho$ Density of air

$p \quad$ Cushion pressure

$H_{T} \quad$ Mean total pressure at nozzle exit

$M$ Mass flow rate of jet at nozzle exit

$\Delta M$ Mass flow rate of air fed into cushion

$u \quad$ Mean jet velocity at nozzle exit

$u_{j} \quad$ Local velocity of fluid in jet

$J$ Momentum flux of jet

$R \quad$ Mean radius of curvature of jet

$C_{p} \quad C_{p}=p / o u^{2}$

* Faculty of Engineering, Kumamoto University

** ACVs means "Air Cushion Vehicles". 
$\sigma \quad$ Empirical constant for balanced jet*

$H$ Empirical constant for balanced jet*

$\kappa \quad$ Empirical constant for unbalanced jet*

$m_{p}$ Mass flow coefficient

$m_{h}$ Mass flow coefficient

$m_{\theta}$ Mass flow coefficient

$\varphi \quad$ Parameter involved in the empirical formulas*

$T$ Parameter involved in the empirical formulas*

\section{Semi-empirical Formulas}

\subsection{Balanced Condition}

From the theoretical analysis, the following formulas were derived in Ref. 2).

$$
\begin{aligned}
& C_{p} \equiv \frac{p}{\rho u^{2}}=\frac{3(\pi-\theta-\varphi)}{\sigma} \frac{T^{2}}{1-T^{2}} \\
& \frac{h}{t}=\frac{\cos \theta+\cos \varphi}{C_{p}}+\frac{H}{3} \frac{\cos \varphi}{T^{2}} \\
& \cos \varphi=\frac{T}{2}\left(3-T^{2}\right)
\end{aligned}
$$

where $p, \rho, u, \theta, h$ and $t$ are the mean cushion pressure, the density of air, the mean jet velocity at the nozzle exit, the jet discharge angle (measured from horizontal downward), the hoverheight and the nozzle thickness, respectively. $\sigma$ and $H$ are empirical constants to be derived from experiments. By the elimination of $T$ and $\varphi$ among Eqs. (1) to (3), we have the relation among $C_{p}, h / t$ and $\theta$. It is to be noted that the above formulas are not applicable when $h / t$ is very small.

\subsection{Unbalanced Condition}

The ratio of the mass flow rate into the cushion $\Delta M$ to the total mass flow rate of the jet at the nozzle exit $M$ is represented as ${ }^{1)}$

$$
\frac{\Delta M}{M}=-m_{p} \delta C_{p}-m_{h} \delta\left(\frac{h}{t}\right)-m_{\theta} \delta \theta
$$

where $\delta C_{p}, \delta(h / t)$ and $\delta \theta$ represent the small variations of $C_{p}, h / t$ and $\theta$ from the balanced condition and

$$
\begin{aligned}
& m_{p}=\frac{1}{M_{e}}\left[N_{e}\left(\frac{h}{t}-\frac{H}{3} \cos \varphi\right)_{e}-\left(\frac{1-T^{2}}{4 C_{p}}\right)_{e}\right] \\
& m_{h}=\frac{N_{e}}{M_{e}} C_{p_{e}} \\
& m_{\theta}=\frac{1}{M_{e}}\left[N_{e}\left(\frac{H}{\sigma} \cos \varphi+\sin \theta\right)-\frac{3 T^{2}}{4 \sigma C_{p}}+\frac{1-T^{2}}{4 \sigma}\left(N_{e} \frac{4}{3} \frac{H}{T^{2}} C_{p} \cos \varphi-1\right) \frac{d \sigma}{d \theta}\right]_{e} \\
& M_{e}=1-\kappa N_{e}\left(\frac{C_{p} \cos \varphi}{3 T^{2}}\right)_{e} \\
& N_{e}=\left[\frac{\frac{1}{3 T} \frac{\sin \varphi}{1-T^{2}}+\frac{3 T^{2}}{4 \sigma C_{p}}}{\left(\frac{H C_{p}}{3 T^{2}}+1\right) \sin \varphi+\frac{H}{\sigma} \cos \varphi}\right]_{e} .
\end{aligned}
$$

The subscript " $e$ " in the above formulas means the values at the balanced condition. For the given

* Originally, these quantities have their own physical meanings. (See Ref. 1), 2))

$\dagger$ In the original theory, the last term of (1) represents $p t / J$, where $J$ is the momentum flux of jet. However, in the experimental determination of $\sigma$ and $H, J$ is approximated by $\rho t u^{2}$. The same is true for the right hand side of (4) which originally represents $\Delta M / \sqrt{\rho J t}$. 
values of $(h / t)_{e}$ and $\theta_{e}, T_{e}, \varphi_{e}$ and $C_{p_{e}}$ are obtained by Eqs. (1) to (3). The empirical constant $\kappa$ is to be determined experimentally. These formulas were originally obtained by theoretical analysis.1)

The above formulas do not give any knowledge about the jet total pressure at the nozzle exit. This will be done approximately by the following consideration. The equation of motion of small element of fluid in jet is written as

$$
\frac{d p}{\rho u j^{2}}=\frac{d Y}{R}
$$

tog ether with the boundary conditions

$$
p=0 \quad \text { at } \quad Y=-\frac{t}{2}, \quad p=p \quad \text { at } \quad Y=\frac{t}{2}
$$

where $Y$ axis is taken normal to the jet centerline (where $Y=0$ ) and $d p$ is the increments of the static pressure in $Y$ direction. We may now assume the potential flow at the nozzle exit; indeed, the potential core is considered to exist there. Applying Bernoulli's theorem, the solution of Eq. (10) is written as

$$
\frac{p}{H_{T}}=1-e^{-(2 t / R)}=1-e^{-(2 p t / J)}
$$

where $H_{T}$ is the total pressure of the jet which is assumed uniform over the nozzle exit. The Eq. (11) holds in the unbalanced condition as well as in the balanced condition.

\section{Comparison with Experimental Results}

A series of experiments to examine the semi-empirical formulas for the balanced jet was already made and reported in Ref. 2). A study for the unbalanced jet was later reported in the previous paper. ${ }^{1)}$ The experiments in Ref. 1), however, were limited to the peripheral jet with the discharge angle of $30^{\circ}$. Further experiments have been made including the discharge angles of $45^{\circ}$ and $60^{\circ}$ to cover wider range and to re-examine the proposed method. The results will be reported in this section. For the method of experiments, Ref. 1) and 2) are to be referred to.

\subsection{Balanced Condition}

The values of $\sigma$ and $d \sigma / d \theta$ which have been obtained from the previous investigations ${ }^{1), 2)}$ are presented in Table 1. It was shown in Ref. 1) that, fixing the values of $\sigma$ in this way, $H$ varies slightly from model to model. The experimental points are plotted in Fig. 1 together with the results of the calculation which are made by Eqs. (1) to (3), using the values of $\sigma$ given in Table 1 and with the suitable choice of $H$.

\begin{tabular}{c|c|c} 
Table 1 & Values of $\sigma$ and $d \sigma / d \theta$ \\
\hline$\theta$ & $\sigma$ & $\begin{array}{c}d \sigma / d \theta \\
(1 / \text { radian })\end{array}$ \\
\hline 30 & 0.50 & 4.37 \\
35 & 0.93 & 5.12 \\
40 & 1.40 & 5.95 \\
45 & 2.00 & 6.96 \\
50 & 2.60 & 8.00 \\
55 & 3.38 & 9.15 \\
60 & 4.20 & 10.33 \\
\hline
\end{tabular}

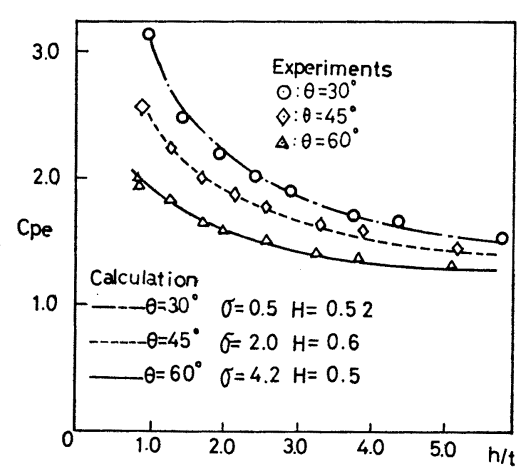

Fig. $1 C_{p}$ in balanced condition

3.2 Unbalanced Condition

The experimental points are plotted in Fig. 2 to Fig. 25 together with the results of calculation. 


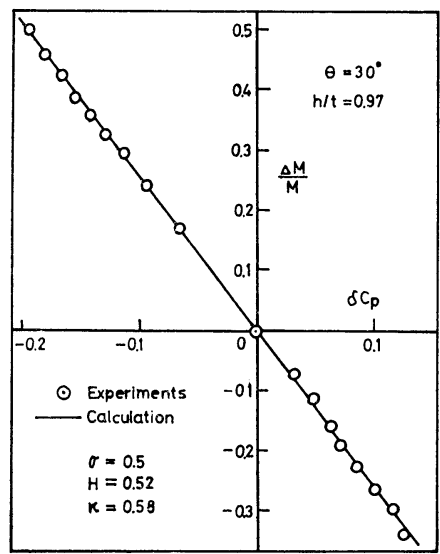

Fig. 2 Mass flow rate into cushion $\left(\theta=30^{\circ}\right)$

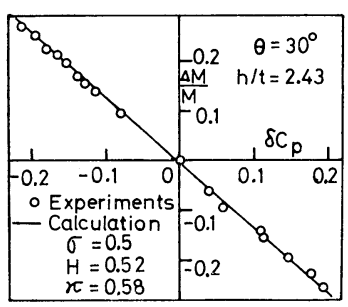

Fig. 5 Mass flow rate into cushion $\left(\theta=30^{\circ}\right)$

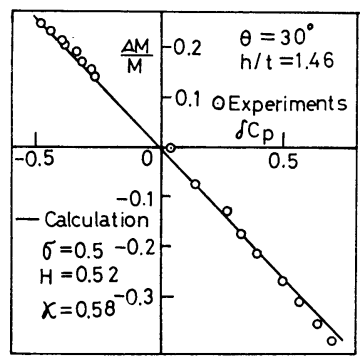

Fig. 3 Mass flow rate into cushion $\left(\theta=30^{\circ}\right)$

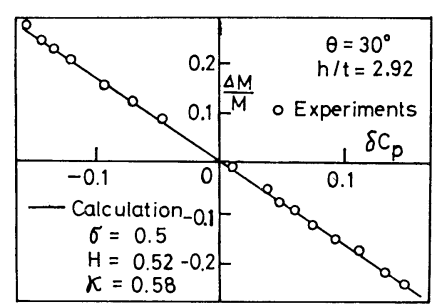

Fig. 6 Mass flow rate into cushion $\left(\theta=30^{\circ}\right)$

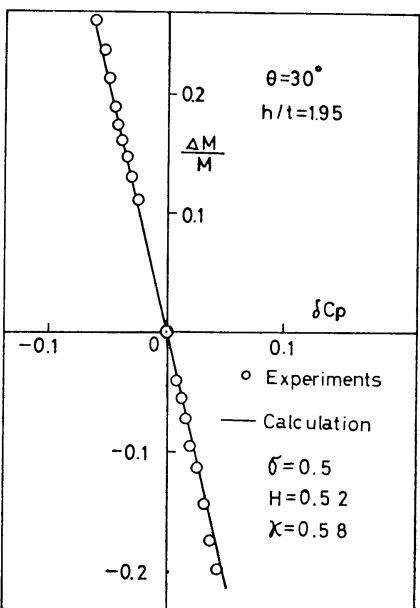

Fig. 4 Mass flow rate into cushion $\left(\theta=30^{\circ}\right)$

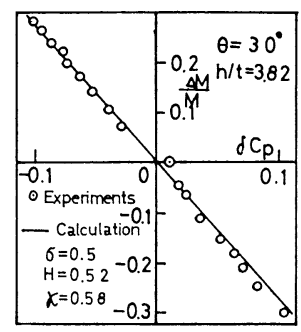

Fig. 7 Mass flow rate into cushion $\left(\theta=30^{\circ}\right)$

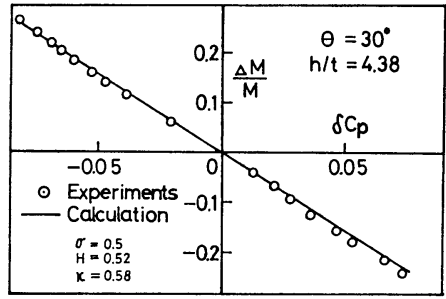

Fig. 8 Mass flow rate into cushion $\left(\theta=30^{\circ}\right)$

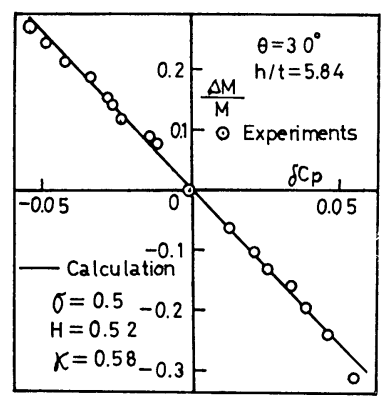

Fig. 9 Mass flow rate into cushion $\left(\theta=30^{\circ}\right)$

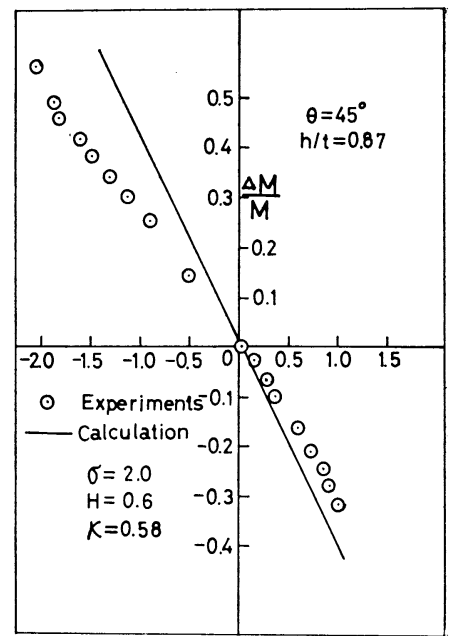

Fig. 10 Mass flow rate into cushion $\left(\theta=45^{\circ}\right)$ 


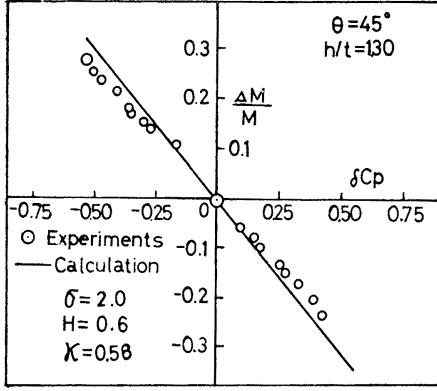

Fig. 11 Mass flow rate into cushion $\left(\theta=45^{\circ}\right)$

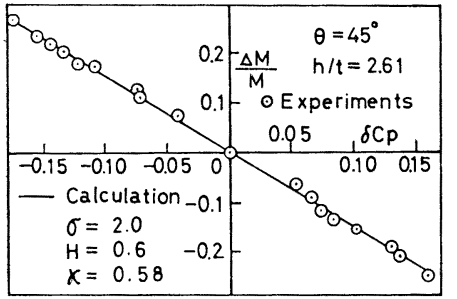

Fig. 14 Mass flow rate into cushion $(\theta=45)$

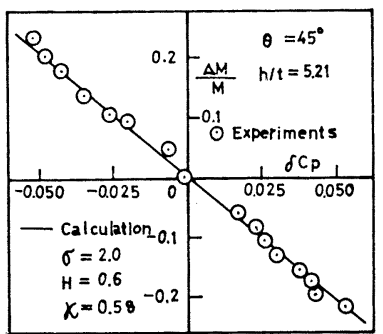

Fig. 17 Mass flow rate into cushion $\left(\theta=45^{\circ}\right)$

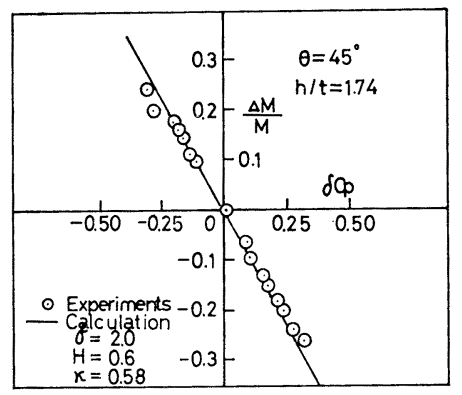

Fig. 12 Mass flow rate into cushion $\left(\theta=45^{\circ}\right)$

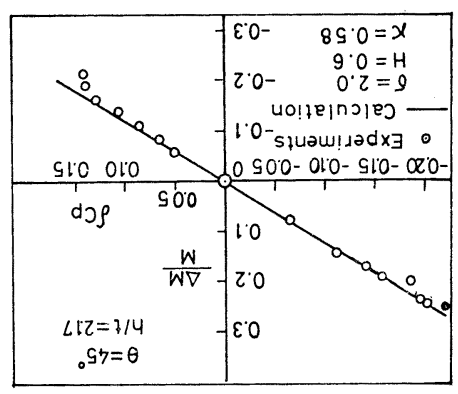

Fig. 13 Mass flow rate into cushion $\left(\theta=45^{\circ}\right)$

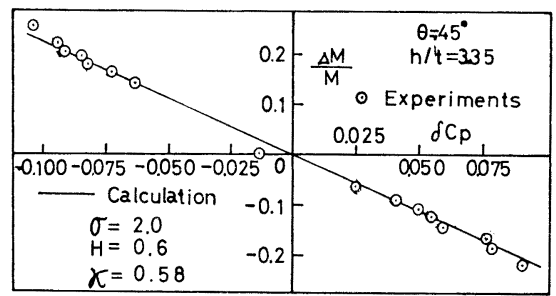

Fig. 15 Mass flow rate into cushion $\left(\theta=45^{\circ}\right)$

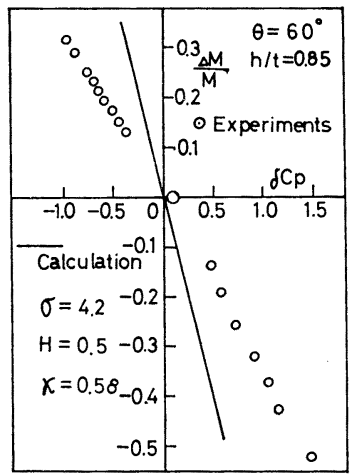

Fig. 18 Mass flow rate into cushion $\left(\theta=60^{\circ}\right)$

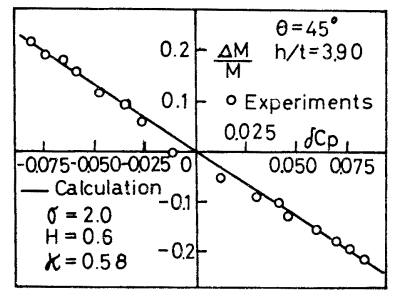

Fig. 16 Mass flow rate into cushion $\left(\theta=45^{\circ}\right)$

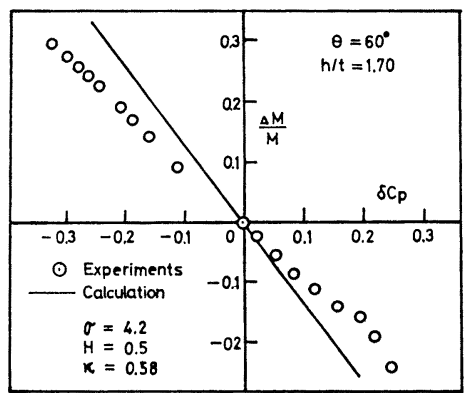

Fig. 20 Mass flow rate into cushion $\left(\theta=60^{\circ}\right)$

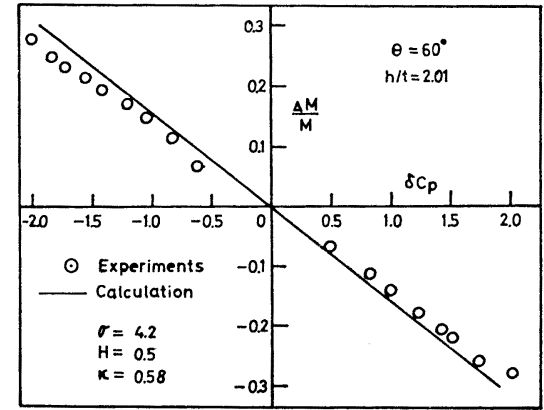

Fig. 21 Mass flow rate into cushion $\left(\theta=60^{\circ}\right)$ 


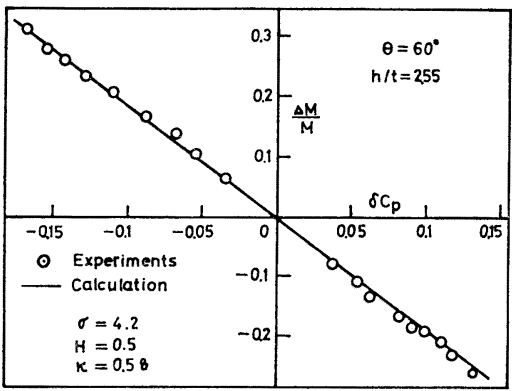

Fig. 22 Mass flow rate into cushion $\left(\theta=60^{\circ}\right)$

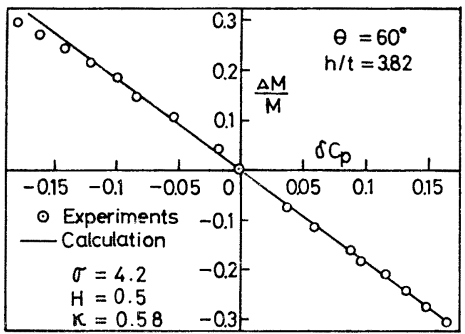

Fig. 24 Mass flow rate into cushion $\left(\theta=60^{\circ}\right)$

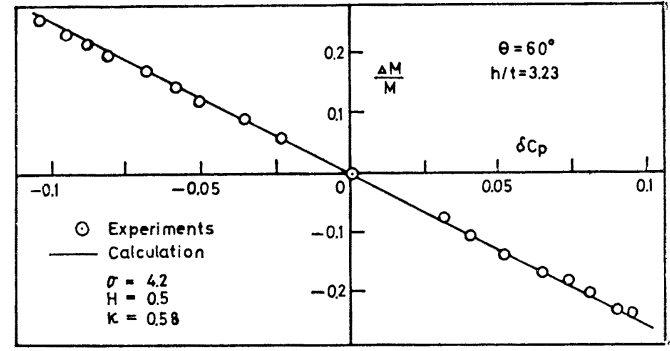

Fig. 23 Mass flow rate into cushion $\left(\theta=60^{\circ}\right)$

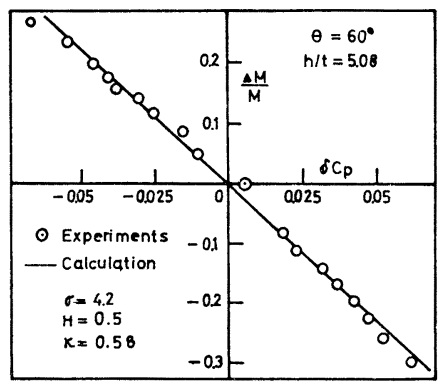

Fig. 25 Mass flow rate into cushion $\left(\theta=60^{\circ}\right)$

The Eqs. (5) to (9) together with the values of $\sigma$ and $d \sigma / d \theta$ given in Table 1 and $H$ obtained for the balanced jet (Fig. 1) are used in calculation. The empirical constant $\kappa$ is taken as 0.58 , the value obtained in the previous investigation. ${ }^{1)}$ Excepting small $h / t$ ratios, the calculations agree well with the experiments. The discrepancies in the range of small $h / t$ ratios become larger as the discharge angle increases. Particularly for the range of small $h / t$ ratios, the slopes of the experimental points at $\Delta M / M=0$; i.e., $-m_{p}$, are read from Figs. 2 to 25 , and shown in Fig. 26 . We see that an abrupt change in $m_{p}$ occurs below $h / t=2$ and that it is significant for $\theta=60^{\circ}$. In order to explain this phenomenon, however, further investigations may be required.

\subsection{Mean Total Pressure}

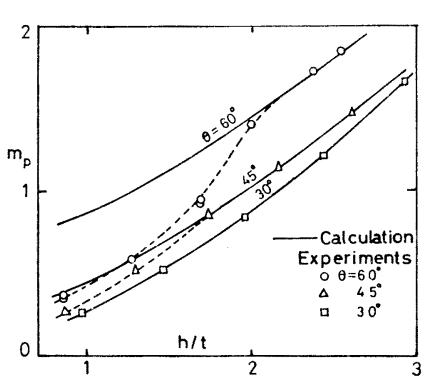

Fig. $26 m_{p}$ in the range of small $h / t$.

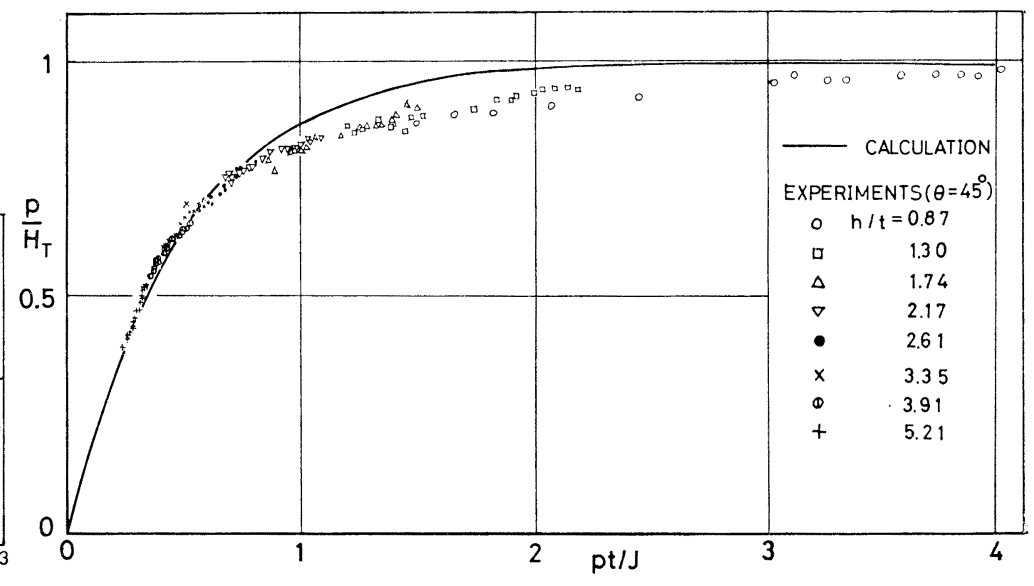

Fig. 27 Mean total pressure at nozzle exit. 
The mean total pressure at nozzle exit is plotted in Fig. 27 together with the theoretical curve obtained from Eq. (11). It is to be noted that, in the analysis of experimental results, $\rho t u^{2}$ is taken in place of J. Although the agreement is not very good, Eq. (11) may be approximately used for the practical purposes.

\section{Numerical Tables}

\subsection{Results obtained from the Preceding Studies}

From the preceding studies including those in Ref. 1) and 2), the following results have been obtained.

(1) Balanced condition.... By suitable choice of $\sigma$ and $H$, the theoretical formulas Eqs. (1) to (3) give fairly good results. However, there exist small differences in the jet performance from model to model mainly due to the secondary effects, such as nozzle configurations, the way of air supply, etc. By fixing $\sigma$ as shown in Table 1, the differences are practically corrected by the different choice of $H$. Actually the value of $H$ seems to range from 0.3 to 0.6 .1$)$ The value of $H=0.45$ may be recommended for rough estimations.

(2) Unbalanced condition.... Once the values of $\sigma$ and $H$ are obtained for the balanced condition, Eqs. (5) to (9) enable us to perform the calculations for unbalanced jets. Except for small $h / t$ and large $\theta$, the fairly good results are obtained.

\subsection{Corrections for the small values of $h / t$ and large $\theta$}

We have

$$
C_{p}=C_{p}\left(\frac{\Delta M}{M}, \frac{h}{t}, \theta\right)
$$

where

$$
C_{p}\left(0, \frac{h}{t}, \theta\right) \equiv C_{p_{e}}\left(\frac{h}{t}, \theta\right)
$$

Putting

$$
C_{p_{1}}\left(\frac{\Delta M}{M}, \frac{h}{t}, \theta\right)=C_{p}\left(\frac{\Delta M}{M}, \frac{h}{t}, \theta\right)-C_{p_{e}}\left(\frac{h}{t}, \theta\right)
$$

we hàve

$$
C_{p_{1}}\left(0, \frac{h}{t}, \theta\right) \equiv 0
$$

and

$$
C_{p}\left(\frac{\Delta M}{M}, \frac{h}{t}, \theta\right)=C_{p_{e}}\left(\frac{h}{t}, \theta\right)+C_{p_{1}}\left(\frac{\Delta M}{M}, \frac{h}{t}, \theta\right) .
$$

From Eq. (16) we have

$$
\delta C_{p}=\left[\begin{array}{c}
\partial C_{p_{e}} \\
\partial(h / t)
\end{array}\right]_{e} \delta\left(\frac{h}{t}\right)+\left(\frac{\partial C_{p_{e}}}{\partial \theta}\right)_{e} \delta \theta+\left[\frac{\partial C_{p_{1}}}{\partial(\Delta M / M)}\right]_{e} \delta\left(\frac{\Delta M}{M}\right)+\left[\frac{\partial C_{p_{1}}}{\partial(h / t)}\right]_{e} \delta\left(\frac{h}{t}\right)+\left(\frac{\partial C_{p_{1}}}{\partial \theta}\right)_{e} \delta \theta \text {. }
$$

The last two terms of Eq. (17) vanish and

$$
\left[\frac{\partial C_{p_{1}}}{\partial(\Delta M / M)}\right]_{e}=-\frac{1}{m_{p}}
$$

From Eq. (17) and Eq. (18), we obtain

$$
\frac{\Delta M}{M}=-m_{p} \delta C_{p}+m_{p}\left[\frac{\partial C_{p_{e}}}{\partial(h / t)}\right]_{e} \delta\left(\frac{h}{t}\right)+m_{p}\left(\frac{\partial C_{p_{e}}}{\partial \theta}\right)_{e} \delta \theta .
$$

Hence we have

$$
m_{p}\left[\frac{\partial C_{p_{e}}}{\partial(h / t)}\right]_{e}=-m_{h}, \quad m_{p}\left(\frac{\partial C_{p_{e}}}{\partial \theta}\right)_{e}=-m_{\theta}
$$


From the preceding investigations, it has been known that the proposed formulas give correct $\left[\partial C_{p_{e}} / \partial(h / t)\right]_{e}$ and $\left(\partial C_{p_{e}} / \partial \theta\right)_{e}$ in so far as suitable choice are made for $\sigma$ and $H$. Hence we can see now that the correction necessary to obtain the values of $m_{p}, m_{h}$ and $m_{\theta}$ is that to $m_{p}$ only. From Figs. 2 to 25 , we see that the calculated $m_{p}$ is larger than actual value in the range of small $h / t$ and large $\theta$. The correction factor $\alpha=\left(m_{p}\right)_{\text {actual }} /\left(m_{p}\right)_{\text {calculation }}$ is derived from Fig. 26 . The results of calculation, which are made by means of Eqs. (1) to (9) together with the values of $\sigma$ and $d \sigma / d \theta$ give in Table 1 and are corrected by $\alpha$, are shown in Table 2 to 5 . It is required only to know the value of $H$ for a particular model. In case that it is not known, the value of $H=0.45$ may be recommended. $C_{p}, m_{p}, m_{h}$ and $m_{\theta}$ for $H=0.45$ are shown graphically in Figs. 28 to 31 .

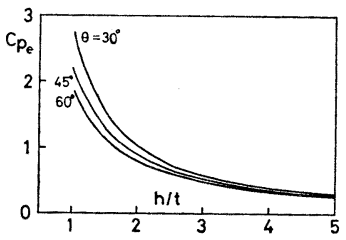

Fig. $28 C_{p}$ in balanced condition $(H=0.45)$

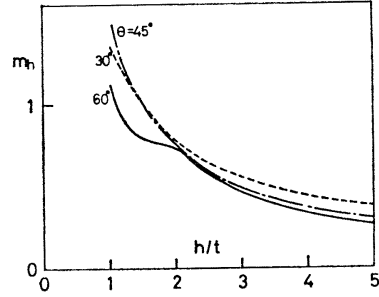

Fig. $30 m_{h}(H=0.45)$

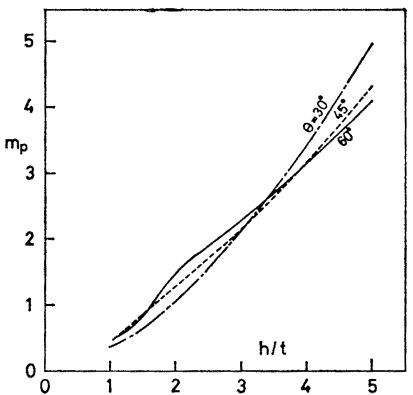

Fig. $29 m_{p}(H=0.45)$

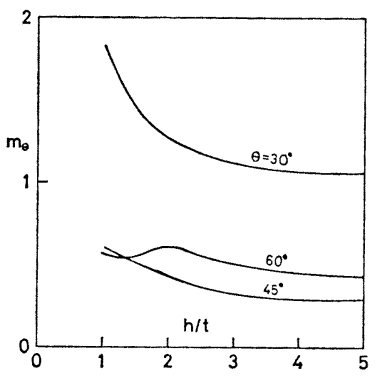

Fig. $31 m_{\theta}(H=0.45)$

Table 2

\begin{tabular}{|c|c|c|c|c|c|c|c|c|c|c|c|c|}
\hline \multirow{3}{*}{$\begin{array}{r}\theta \\
h / t\end{array}$} & \multicolumn{12}{|c|}{ Values of $C_{p_{e}}$} \\
\hline & \multicolumn{4}{|c|}{$\theta=30^{\circ}$} & \multicolumn{4}{|c|}{$\theta=45^{\circ}$} & \multicolumn{4}{|c|}{$\theta=60^{\circ}$} \\
\hline & 0.3 & 0.4 & 0.5 & 0.6 & 0.3 & 0.4 & 0.5 & 0.6 & 0.3 & 0.4 & 0.5 & 0.6 \\
\hline 1 & 2.1951 & 2.5644 & 2.9942 & 3.4931 & 1.9266 & 2.0958 & 2.2829 & 2.4900 & 1.6902 & 1.8020 & 1.9241 & 2.0576 \\
\hline 1.5 & 1.2595 & 1.4271 & 1.6161 & 1.8289 & 1.1598 & 1.2387 & 1.3236 & 1.4149 & 1.0414 & 1.0931 & 1.1480 & 1.2064 \\
\hline 2 & 0.8601 & 0.9574 & 1.0652 & 1.1846 & 0.8112 & 0.8579 & 0.9074 & 0.9600 & 0.7389 & 0.7695 & 0.8016 & 0.8352 \\
\hline 2.5 & 0.6441 & 0.7084 & 0.7790 & 0.8561 & 0.6153 & 0.6465 & 0.6794 & 0.7140 & 0.5656 & 0.5861 & 0.6074 & 0.6297 \\
\hline 3 & 0.5107 & 0.5568 & 0.6069 & 0.6613 & 0.4913 & 0.5138 & 0.5374 & 0.5621 & 0.4542 & 0.4690 & 0.4844 & 0.5003 \\
\hline 3.5 & 0.4209 & 0.4557 & 0.4934 & 0.5340 & 0.4063 & 0.4234 & 0.4413 & 0.4599 & 0.3770 & 0.3883 & 0.3999 & 0.4119 \\
\hline 4 & 0.3566 & 0.3841 & 0.4136 & 0.4452 & 0.3449 & 0.3584 & 0.3724 & 0.3870 & 0.3207 & 0.3296 & 0.3387 & 0.3482 \\
\hline 4.5 & 0.3086 & 0.3309 & 0.3547 & 0.3801 & 0.2986 & 0.3095 & 0.3209 & 0.3327 & 0.2779 & 0.2851 & 0.2925 & 0.3002 \\
\hline 5 & 0.2715 & 0.2899 & 0.3096 & 0.3306 & 0.2625 & 0.2716 & 0.2811 & 0.2908 & 0.2444 & 0.2504 & 0.2565 & 0.2629 \\
\hline
\end{tabular}


Table 3

\begin{tabular}{|c|c|c|c|c|c|c|c|c|c|c|c|c|}
\hline \multirow{3}{*}{$\begin{array}{r}\theta \\
h / t\end{array}$} & \multicolumn{12}{|c|}{ Values of $m_{p}$} \\
\hline & \multicolumn{4}{|c|}{$\theta=30^{\circ}$} & \multicolumn{4}{|c|}{$\theta=45^{\circ}$} & \multicolumn{4}{|c|}{$\theta=60^{\circ}$} \\
\hline & 0.3 & 0.4 & 0.5 & 0.6 & 0.3 & 0.4 & 0.5 & 0.6 & 0.3 & 0.4 & 0.5 & 0.6 \\
\hline 1 & 0.7241 & 0.4357 & 0.2911 & 0.2069 & 0.6563 & 0.5117 & 0.4092 & 0.3334 & 0.5922 & 0.4981 & 0.4236 & 0.3634 \\
\hline 1.5 & 1.2571 & 0.8099 & 0.5668 & 0.4177 & 1.1657 & 0.9570 & 0.7987 & 0.6753 & 1.0126 & 0.8890 & 0.7854 & 0.6976 \\
\hline 2 & 1.9152 & 1.2804 & 0.9204 & 0.6928 & 1.6687 & 1.4055 & 1.1991 & 1.0338 & 1.7447 & 1.5635 & 1.4076 & 1.2723 \\
\hline 2.5 & 2.6813 & 1.8368 & 1.3444 & 1.0269 & 2.1455 & 1.8363 & 1.5888 & 1.3870 & 2.1957 & 1.9925 & 1.8148 & 1.6584 \\
\hline 3 & 3.5449 & 2.4716 & 1.8333 & 1.4159 & 2.6672 & 2.3088 & 2.0175 & 1.7771 & 2.6151 & 2.3936 & 2.1977 & 2.0237 \\
\hline 3.5 & 4.4982 & 3.1790 & 2.3828 & 1.8563 & 3.2300 & 2.8198 & 2.4828 & 2.2020 & 3.0601 & 2.8189 & 2.6038 & 2.4112 \\
\hline 4 & 5.5352 & 3.9545 & 2.9890 & 2.3451 & 3.8310 & 3.3670 & 2.9825 & 2.6596 & 3.5287 & 3.2668 & 3.0317 & 2.8199 \\
\hline 4.5 & 6.6510 & 4.7941 & 3.6491 & 2.8798 & 4.4682 & 3.9486 & 3.5150 & 3.1486 & 4.0195 & 3.7361 & 3.4805 & 3.2490 \\
\hline 5 & 7.8414 & 5.6947 & 4.3603 & 3.4584 & 5.1397 & 4.5629 & 4.0787 & 3.6674 & 4.5313 & 4.2259 & 3.9493 & 3.6977 \\
\hline
\end{tabular}

Table 4

\begin{tabular}{|c|c|c|c|c|c|c|c|c|c|c|c|c|}
\hline & \multicolumn{12}{|c|}{ Values of $m_{h}$} \\
\hline$\theta$ & \multicolumn{4}{|c|}{$\theta=30^{\circ}$} & \multicolumn{4}{|c|}{$\theta=45^{\circ}$} & \multicolumn{4}{|c|}{$\theta=60^{\circ}$} \\
\hline$h / t \quad H$ & 0.3 & 0.4 & 0.5 & 0.6 & 0.3 & 0.4 & 0.5 & 0.6 & 0.3 & 0.4 & 0.5 & 0.6 \\
\hline 1 & 2.2222 & 1.6571 & 1.3674 & 1.1962 & 1.5929 & 1.4091 & 1.2808 & 1.1886 & 1.2019 & 1.1202 & 1.0585 & 1.0120 \\
\hline 1.5 & 1.4182 & 1.0867 & 0.9019 & 0.7855 & 1.1226 & 1.0145 & 0.9324 & 0.8686 & 0.8377 & 0.7922 & 0.7547 & 0.7235 \\
\hline 2 & 1.0778 & 0.8371 & 0.6972 & 0.6066 & 0.8395 & 0.7665 & 0.7089 & 0.6626 & 0.7703 & 0.7335 & 0.7020 & 0.6748 \\
\hline 2.5 & 0.8864 & 0.6942 & 0.5797 & 0.5042 & 0.6530 & 0.6001 & 0.5574 & 0.5223 & 0.5965 & 0.5704 & 0.5476 & 0.5276 \\
\hline 3 & 0.7623 & 0.6005 & 0.5024 & 0.4369 & 0.5386 & 0.4972 & 0.4632 & 0.4350 & 0.4775 & 0.4581 & 0.4408 & 0.4254 \\
\hline 3.5 & 0.6746 & 0.5337 & 0.4472 & 0.3889 & 0.4611 & 0.4270 & 0.3988 & 0.3751 & 0.3991 & 0.3837 & 0.3699 & 0.3575 \\
\hline 4 & 0.6088 & 0.4834 & 0.4055 & 0.3527 & 0.4050 & 0.3761 & 0.3519 & 0.3315 & 0.3436 & 0.3309 & 0.3195 & 0.3092 \\
\hline 4.5 & 0.5575 & 0.4439 & 0.3728 & 0.3242 & 0.3624 & 0.3373 & 0.3161 & 0.2981 & 0.3022 & 0.2915 & 0.2818 & 0.2729 \\
\hline 5 & 0.5162 & 0.4119 & 0.3462 & 0.3011 & 0.3290 & 0.3067 & 0.2878 & 0.2717 & 0.2702 & 0.2609 & 0.2525 & 0.2448 \\
\hline
\end{tabular}

Table 5

\begin{tabular}{|c|c|c|c|c|c|c|c|c|c|c|c|c|}
\hline \multirow[b]{2}{*}{$\theta$} & \multicolumn{12}{|c|}{ Values of $m_{\theta}$} \\
\hline & \multicolumn{4}{|c|}{$\theta=30^{\circ}$} & \multicolumn{4}{|c|}{$\theta=45^{\circ}$} & \multicolumn{4}{|c|}{$\theta=60^{\circ}$} \\
\hline$h / t \quad H$ & 0.3 & 0.4 & 0.5 & 0.6 & 0.3 & 0.4 & 0.5 & 0.6 & 0.3 & 0.4 & 0.5 & 0.6 \\
\hline 1 & 1.5303 & 1.7595 & 1.8836 & 1.9637 & 0.5386 & 0.5856 & 0.6208 & 0.6486 & 0.5838 & 0.5688 & 0.5575 & 0.5488 \\
\hline 1.5 & 1.0349 & 1.3389 & 1.5106 & 1.6211 & 0.4153 & 0.4848 & 0.5392 & 0.5829 & 0.5438 & 0.5443 & 0.5449 & 0.5456 \\
\hline 2 & 0.8324 & 1.1556 & 1.3441 & 1.4671 & 0.3251 & 0.3972 & 0.4551 & 0.5026 & 0.6053 & 0.6129 & 0.6197 & 0.6258 \\
\hline 2.5 & 0.7324 & 1.0602 & 1.2555 & 1.3845 & 0.2656 & 0.3339 & 0.3897 & 0.4361 & 0.5437 & 0.5538 & 0.5628 & 0.5709 \\
\hline 3 & 0.6804 & 1.0072 & 1.2050 & 1.3369 & 0.2341 & 0.2985 & 0.3517 & 0.3965 & 0.4941 & 0.5047 & 0.5142 & 0.5229 \\
\hline 3.5 & 0.6546 & 0.9780 & 1.1759 & 1.3089 & 0.2183 & 0.2790 & 0.3296 & 0.3725 & 0.4629 & 0.4733 & 0.4828 & 0.4915 \\
\hline 4 & 0.6446 & 0.9631 & 1.1600 & 1.2931 & 0.2121 & 0.2694 & 0.3175 & 0.3586 & 0.4431 & 0.4531 & 0.4622 & 0.4706 \\
\hline 4.5 & 0.6445 & 0.9575 & 1.1526 & 1.2852 & 0.2122 & 0.2663 & 0.3121 & 0.3514 & 0.4309 & 0.4403 & 0.4490 & 0.4569 \\
\hline 5 & 0.6511 & 0.9583 & 1.1511 & 1.2827 & 0.2165 & 0.2678 & 0.3114 & 0.3489 & 0.4240 & 0.4328 & 0.4409 & 0.4484 \\
\hline
\end{tabular}




\section{Concluding Remarks}

(1) Semi-empirical formulas for estimation of ACV jet performance which the author previously proposed are re-examined over wider range of operating conditions and numerical tables are given so that they may be used for practical purposes.

(2) The proposed method requires to know the value of an empirical constant $H$ before hand for a particular model. This is the consequence of the fact that the theoretical analysis on which the proposed empirical formulas are based does not involve the considerations on such secondary effects as the configuration of nozzle and ducts, the way of air supply, etc. To consider such effects is however very difficult.

(3) It is to be noted that the validity of the proposed formulas for $m_{h}$ and $m_{\theta}$ is ascertained by the consideration on their theoretical relations to $C_{p_{e}}$ and $m_{p}$ given by Eq. (20), while the formulas for $C_{p_{e}}$ and $m_{p}$ are examined directly by the experiments. Further experimental investigations directly to measure $m_{h}$ and $m_{\theta}$ may be desirable. Some of those methods are suggested as follows.

(i) The direct measurements of $m_{h}$ and $m_{\theta}$ in case that $\delta C_{p}=\delta \theta=0$ and $\delta C_{p}=\delta(h / t)=0$ respectively.

(ii) The comparison of the calculated $m_{h}$, say, with that obtained using the known value of $m_{p}$ and the experimental result in case that $\delta \theta=0$. Further comparison of the calculated $m_{\theta}$ with that obtained using the known values of $m_{p}$ and $m_{h}$ and the experimental result in case that none of $\delta C_{p}, \delta(h / t)$ and $\delta \theta$ is zero.

However, in so far as we deal with such small changes as to be treated by linear approximation, the proposed method seems to be sufficient.

\section{Acknowledgement}

The author wishes to express his appreciation to Mr. K. Harada, Mr. S. Okamura and Mr. A. Shigaki, who are the former students of Faculty of Engineering, Kumamoto University, for their cooperation in performing the experiments. Numerical calculation was made by means of FACOMI 231 Type digital computer at the Computation Center of Kumamoto University and he is indebted to all the members of the staff of the Center.

\section{References}

1) H. Matsuo: A Study of the Peripheral Jet of the Air Cushion Vehicles, Jour. Soc. Naral Architects, Japan, Vol. 127, (1970).

2) H. Matsuo: A Method for Predicting GEM Cushion Pressure, Jour. Soc. Naval Architects, Japan, Vol. 119, (1966). 